\title{
AN sS MODEL WITH ADVERSE SELECTION
}

\author{
Christopher L. House \\ John V. Leahy \\ Working Paper 8030 \\ http://www.nber.org/papers/w8030

\section{NATIONAL BUREAU OF ECONOMIC RESEARCH 1050 Massachusetts Avenue \\ Cambridge, MA 02138 \\ December 2000}

We would like to thank Guillermo Caruana, Igal Hendel and Jon Willis for helpful comments and suggestions. Leahy thanks the National Science Foundation for financial support. The views expressed in this paper are those of the authors and not necessarily those of the National Bureau of Economic Research.

(C) 2000 by Christopher L. House and John V. Leahy. All rights reserved. Short sections of text, not to exceed two paragraphs, may be quoted without explicit permission provided that full credit, including (C) notice, is given to the source. 
An sS Model with Adverse Selection

Christopher L. House and John V. Leahy

NBER Working Paper No. 8030

December 2000

JEL No. D82, E21, L15

\section{$\underline{\text { ABSTRACT }}$}

We present a model of the market for used cars in which agents face a fixed cost of adjustment, the magnitude of which depend on the degree of adverse selection in the secondary market. We find that, unlike typical models, the sS bands in our model contract as the variance of the shock process increases. We also analyze a dynamic version of the model in which agents are allowed to make decisions that are conditional of the age of a used car. We find that, as a car ages, the lemons problem tends to decline in importance, and the sS bands contract.

Christopher L. House

Department of Economics

Boston University

270 Bay State Road

Boston, MA 02115
John V. Leahy

Department of Economics

Boston University

270 Bay State Road

Boston, MA 02115

and NBER

jleahy@bu.edu 


\section{Introduction}

Given that consumers do not replace durables every day, it has become common to assume that purchases of durables are subject to fixed costs of adjustment. Among the fixed costs most often cited are "lemons costs." 1 These costs arise because adverse selection in the secondary market reduces the price of a durable, and thereby discourages trade (Akerlof [1970]). The size of this cost depends on the distribution of quality in the secondary market, and this distribution depends on the sales decisions of consumers. In spite of the obvious endogeneity of such costs, models of durables typically assume that the fixed costs of adjustment are exogenous.

The model that we present is standard except that the adjustment cost arises endogenously from adverse selection in the secondary market. In the model, consumers hold cars of various qualities. Only the owner of a car knows its true quality. There is also a parameter that characterizes the quality of the match between the car and its owner. A good match is more desirable than a poor match. Consumers may sell cars either because the quality is low or because the car is not a good match for their current needs. Because the quality of cars on the secondary market tends to be below that of used cars, holders of relatively good used cars face a cost to selling their car and purchasing a new one. This is the only adjustment cost in the model. The relative proportions of consumers selling on the basis of quality and fit will influence the size of this cost. The greater the quantity of high quality cars on the secondary market, the lower the adjustment cost.

The optimal adjustment policy is a state-contingent $s S$ policy. Contingent on the age and the quality of the car, consumers continue to hold the car if the match parameter lies within some range about the optimum. The limits of this range of inaction, the consumers $s S$ bands, tend to be wider, the higher the quality of the auto. This reflects the adverse selection in the market.

In section 2, we study the interaction between adverse selection and adjustment in a model in which cars last for only two periods. This setting illustrates the strategic complementarity between individual adjustment decisions. If some agents sell their cars in order to improve their match, then the quality of cars in the secondary market improves, the adjustment costs

\footnotetext{
${ }^{1}$ See, for example, Bar-Ilan and Blinder [1992], Caballero [1994], Eberly [1994]. Abel et al. [1996] use lemons effects to motivate investment inertia.
} 
fall, and the incentive to adjust is thereby increased. This feedback between the sales decisions and the adjustment cost leads to the possibility of multiple equilibria. In some equilibria the $s S$ bands are wide, adjustment by owners of high quality cars is rare, and the lemons problem is severe. In others the $s S$ bands are narrow, all types of agent adjust, and the lemons problem is mild.

Our first comparative static result concerns the effect of an increase in the variance of the shock to the match parameter. In $s S$ models with exogenous fixed costs, such an increase would lead agents to widen their bands and to adjust more often. In effect, they divide the cost of the increased variance between larger deviations from the optimal state and more frequent payment of the adjustment cost. In our model, however, there is an additional effect. More frequent adjustment means more agents are selling their cars. This implies that the average quality in the secondary market improves and the adjustment cost falls. Hence the bands may narrow instead.

In Section 3, we extend the life of cars, which introduces additional dynamic elements. Our focus is on the evolution of the $s S$ triggers as cars age. We find that the bands tend to narrow with age, so that adverse selection is initially severe, but lessons with time. This effect arises from two distinct sources. First, if the shocks to the match parameter accumulate over time, then the mismatch between an owner and an auto is greater the longer the car is held. This means that there is greater reason to sell a good old car than a good new car, so that the distribution of quality in the secondary market tends to improve with age. As the distribution of quality improves, the adjustment costs fall, and the $s S$ bands narrow.

The second reason that the bands may narrow with age is that, if trading history is observable, holding a car may serve as a signal that the car is high quality. Since agents with good cars face higher adjustment costs and have wider $s S$ bands, cars that remain unsold are more likely to be of high quality, whereas cars that are sold early are more likely to be lemons.

In both cases, the prospect that the market may improve with time creates a further incentive to wait and sell at a later date. Agents know that as quality in the secondary market improves, so will the price that they can receive for their car. This effect also widens the bands for newer cars.

Together these effects can explain why the lemons problem is more severe for new cars. New cars lose as much as 20 percent of their value as soon as 
they are driven off the lot. ${ }^{2}$ There is understandably considerable reluctance to sell a new car. Few people appear to have similar trouble selling four or five year old cars.

\section{Related Literature}

As the introduction made clear our paper relates to two literatures: $s S$ adjustment and adverse selection. It is somewhat surprising that these literatures have not been brought together earlier, since each in its own way attempts to explain inertia.

In the adverse selection literature, the most closely related paper is that of Hendel and Lizzeri [1999]. They construct a dynamic model with adverse selection in the used car market. ${ }^{3}$ They motivate trade by assuming that agents differ in their taste for quality. They find that some agents refrain from selling high quality used cars due to the cost imposed by adverse selection in the secondary market. They also demonstrate the possibility of multiple equilibria. The main difference between our approach and theirs is that we motivate trade by means of a match parameter that evolves over time. Their taste for quality is an individual fixed effect. They cannot talk about adjustment triggers and targets.

The second literature is the literature on $s S$ adjustment. The $s S$ model was developed by Arrow, Harris, and Marschak [1951] in the context of inventories and extended to the consumption of a durable good by Grossman and Laroque [1991]. There is by now a large literature on durable adjustment. ${ }^{4}$ Most of these models do not contain any equilibrium interactions.

Papers that do allow for equilibrium interactions focus on the determination of the price of the commodity, not the adjustment cost. Among these papers, Stolyarov [1999] is the most closely related to our work. ${ }^{5}$ He considers an environment in which cars depreciate over time and trade is motivated by heterogeneity in the taste for quality. He shows which qualities are produced new, which are traded on secondary markets, and solves for the prices

\footnotetext{
${ }^{2}$ Stiglitz [1997, p. 433].

${ }^{3}$ Blouin [1998] constructs a model along similar lines.

${ }^{4}$ See, for example, Bertola and Cabellero [1990], Bar-Ilan and Blinder [1992], Cabellero [1993], Eberly [1994], Carroll and Dunn [1997], Adda and Cooper [1999], Caplin and Leahy [1999], Leahy and Zeira [1999]

${ }^{5}$ Caplin and Leahy [1999] and Leahy and Zeira [1999] also endogenize prices in an $s S$ model.
} 
that clear secondary markets. He does not, however, incorporate adverse selection. The costs of adjustment in his model are exogenous.

Eisfeldt [1999] constructs a model of an equity market which has aspects of both $s S$ behavior and adverse selection. Agents issue equity for one of two reasons: the agent knows that the project is bad or the agent needs the money. Her model exhibits a financial accelerator. Higher aggregate productivity causes agents to increase the size of their investments which has the effect of increasing the variance of their income. As this variance increases more agents sell claims to high quality projects, which reduces the adverse selection problem and improves the efficiency of the equity market. The fundamental properties of this mechanism are very similar to the mechanism at work in our static model of Section 3. Eisfeldt, however, does not consider multi-period projects and so cannot analyze how the threshold for issuing equity evolves over time.

\section{The Model}

Time is discrete and indexed by $t \in\{0,1, \ldots\}$. There is a continuum of infinitely lived consumers indexed by $i \in[0,1]$, each of whom inelastically demands a single automobile. Consumers care both about the quality of the car that they own and how well it meets their needs. Cars come in two types: good cars and bad cars. Consumers derive greater utility from good cars. Needs are reflected by a match parameter, $z_{i t}$, which summarizes all other motivations for trade beside car quality, including, among other things, tastes, income, and demographics. Each period $t$ each agent $i$ receives utility:

$$
U_{i t}=x_{i t}-z_{i t}^{2}-\varphi_{i t}
$$

Here $x$ is a random variable taking the value 1 if the quality of the car is good and $\phi \in(0,1)$ if the quality is bad, and $\varphi_{i t}$ represents net spending on cars. Consumers discount future payoffs by $\beta$.

Cars last for two periods. After two periods they depreciate completely and the owner must purchase another. Owners of one year old cars may also purchase another car, but to do so they must sell the car that they possess. In any period there are two markets in operation: a market for new cars and a market for one year old cars (used cars).

New cars are supplied by dealers. We do not model the dealers' problem in detail. The only properties that we need are the price of a new car and the 
probability that a new car is good quality. ${ }^{6}$ Let $p_{0}$ denote the exogenous price of a new car, and let $\pi \in(0,1)$ denote the exogenous probability that a new car is good quality. It will also be useful to let $q_{0}=\pi+(1-\pi) \phi$ denote the expected quality of a new car. To ensure that consumers willingly purchase new autos, we assume that $(1+\beta) q_{0}>p_{0}$.

We assume that the quality of any particular car, whether new or used, is the private information of the owner. This gives rise to the adverse selection problem that makes adjustment in this model interesting.

When a consumer purchases a car they choose one that is a good match. Thus a new or used car purchased in period $t$ will have a match parameter $z_{i t}=0$. The match evolves over time, so that if a consumer bought a new car in period $t-1$, the match parameter $z_{i t}$ is a random variable, whose distribution is described by a distribution function $F$ on $R$. We will make assumptions on $F$ as necessary in order to ensure uniqueness, continuity or differentiability of a solution. At this point we only assume that the $z_{i t}$ are independent across time and across agents and that $\int_{-z}^{z} d F<1$ for all $z>0$. This last assumption ensures that some agents receive shocks to their match that are so bad that they adjust under any circumstances.

The assumption that $\int_{-z}^{z} d F<1$ for all $z>0$ guarantees that there is always a positive supply of used cars on the secondary market. It does not, however, ensure that there is positive demand. If the price of new cars is low enough agents may prefer to scrap their used cars and then purchase new ones. We begin with the case in which $p_{0}$ is high enough $\left(p_{0} \geq(1-\phi)(\beta+\pi)\right.$ turns out to be sufficient) that there exists an active secondary market for used cars. We consider an equilibrium with scrapping below.

In period zero, one half of the consumers begin with one year old cars. The rest begin without cars. The timing of moves in each period is as follows. At the beginning of period $t$ holders of one year old cars observe the match parameter $z_{i t}$. Next the markets for new and used cars open simultaneously. After trade is completed, consumers observe the quality of the car that they own (if they do not already know it) and realize utility $U_{i t}$.

Holders of one year old cars choose whether to sell their used cars, while purchasers of cars decide between new and used cars. We look for a stationary symmetric Nash equilibrium as a solution to our model.

\footnotetext{
${ }^{6}$ Given the inelastic demand for autos, the price of new cars will be determined in equilibrium by the marginal cost of production (assuming that the dealers behave competitively).
} 


\subsection{Solution}

Since all consumers purchasing cars are alike, if there is an active secondary market for used cars, consumers who are making adjustments must be indifferent in equilibrium between purchasing a new car and a used car. We use this fact to solve for the optimal adjustment strategies. We solve the model recursively. First, we solve for the optimal adjustment policy of the holder of a one year old car. This determines the average quality of cars in the used car market. We then solve for the price of used cars.

Let $V_{1}(x, z)$ denote the value of an optimal policy for an agent who enters the period holding a one year old car of quality $x$ and a match $z$. The agent decides whether to keep the car or to purchase another car. Since in equilibrium the agent is indifferent between purchasing a new car and used car, we may assume for the purpose of determining the optimal policy that the agent decides to purchase another one year old car. The value function becomes:

$$
V_{1}(x, z)=\max \left\{x-z^{2}+\beta V_{0}, q_{1}+\beta V_{0}\right\}
$$

where $q_{1} \in[\phi, 1]$ is the average quality of cars in the secondary market and $V_{0}$ is the value of purchasing a new car. The first term inside the maximum is the value of holding onto the car. The second is the expected payoff from selling the car buying another used car. Note that the price of used cars does not appear in this second term, since the agent both buys and sells a used car. Regardless of the consumer's decision, the car dies after one period, and the consumer is forced to purchase another car. For simplicity we assume that this is a new car (recall the consumer will be indifferent). Since the continuation payoff is independent of the current choice, the consumer faces what is essentially a static decision: buy or hold depending on the current period's payoff.

Since $q_{1} \in[\phi, 1]$, it follows immediately that holders of lemons always choose to adjust and holders of good used cars adjust if $|z| \geq \bar{z}$ where $^{7}$

$$
\bar{z}=\sqrt{1-q_{1}}
$$

Since all the holders of lemons adjust, we know that the expected quality on the secondary market is less than the expected quality of new cars, $q_{1} \leq q_{0}$. It follows that $\bar{z}>0$. If $F$ places positive probability on the neighborhood

\footnotetext{
${ }^{7}$ For simplicity we assume that agents adjust when indifferent.
} 
$\left(-\sqrt{1-q_{1}}, \sqrt{1-q_{1}}\right)$, then in any equilibrium there will be a positive measure of agents who choose to hold on to their old cars.

In equilibrium, the quality of cars in the secondary market depends on the number of agents holding good used cars that decide to adjust, that is $q_{1}$ depends on $\bar{z}$ and $F$. Let $\lambda_{F}$ denote the proportion of cars in the secondary market that are good quality. Then

$$
\lambda_{F}=\frac{\left(1-\int_{-\bar{z}}^{\bar{z}} d F\right) \pi}{1-\pi \int_{-\bar{z}}^{\bar{z}} d F}
$$

and

$$
q_{1}=\lambda_{F}+\left(1-\lambda_{F}\right) \phi
$$

In order to demonstrate the existence of an equilibrium, it is useful to construct the mapping $T_{F}:[\phi, 1] \rightarrow[\phi, 1]$ as follows. Given $q_{1} \in[\phi, 1]$, equations (1) and (2) pin down $\bar{z}$ and $\lambda_{F}$ recursively. Then given $\lambda_{F}$, equation (3) defines $q_{1}^{\prime} \in[\phi, 1]$. We set $T_{F}\left(q_{1}\right)=q_{1}^{\prime}$. The equilibrium level of $q_{1}$ is then a fixed point of $T_{F}$. Equilibrium levels of $\bar{z}$ and $\lambda_{F}$ follow from equations (1) and $(2)$.

Existence of an equilibrium $q_{1}$ follows from the monotonicity of $T_{F}$ and Tarsky's fixed point theorem. All proofs are contained in the appendix.

Proposition 1: $T_{F}$ is non-decreasing, upper semi-continuous, and has the fixed point property.

Given $\bar{z}, \lambda_{F}$, and $q_{1}$, the price in the second hand market is determined by arbitrage between the new and used car markets:

$$
V_{0}=q_{1}-p_{1}+\beta V_{0}
$$

where $p_{1}$ is the price of a used car. Solving for the price of used cars

$$
p_{1}=q_{1}-(1-\beta) V_{0}
$$

Predictably, the price of used cars is increasing in the quality and decreasing in the value of purchasing new cars.

Finally, we can solve for the value of purchasing a new car:

$$
\begin{gathered}
V_{\mathbf{0}}=\pi\left(1+\beta\left[\alpha q_{1}+(1-\alpha)\left(1-\frac{1}{1-\alpha} \int_{-\bar{z}}^{\bar{z}} z^{2} d F\right)\right]\right) \\
+(1-\pi)\left(\phi+\beta q_{1}\right)+\beta^{2} V_{\mathbf{0}}-p_{\mathbf{0}}
\end{gathered}
$$


where $\alpha=1-\int_{-\bar{z}}^{\bar{z}} d F$. A new car purchased in period $t$ is good with probability $\pi$. With probability $\alpha$ the match worsens in period $t+1$ to the point that the agent sells the car. Given indifference between purchasing a new or a used car, we assume in this case the agent purchases another used car. With probability $1-\alpha$ the agent holds onto the car in $t+1$ and receives the conditional expectation of $x-z^{2}$. The new car is bad with probability $1-\pi$, in which case the agent receives $\phi$ in period $t$ and sells the car in period $t+1$. Whether the new car is good or bad, the price of the car in period $t$ is $p_{0}$ and the agent purchase a new car in period $t+2 . V_{0}>0$ follows from the assumption that $(1+\beta) q_{0}>p_{0}$. Note also that $\frac{1}{1-\alpha} \int_{-\bar{z}}^{\bar{z}} z^{2} d F(z)>0, q_{1}<1$, and $\phi<1$ imply

$$
V_{0}<\frac{1}{1-\beta}-p_{0}
$$

A little algebra shows that $p_{0} \geq(1-\phi)(\beta+\pi)$ is sufficient for $p_{1} \geq 0$ in equation (4).

Together equations (1) through (4) determine $\bar{z}, \lambda_{F}, q_{1}, V_{0}$, and $p_{1}$. This completes the solution to the model.

\subsection{Discussion}

The first thing to notice is that in a lemons model different agents have different agents face different incentives when contemplating adjustment. These incentives are connected to the quality of the car. Owners of higher quality cars face greater costs of adjustment. This contrasts with the fixed costs of adjustment normally imposed by $s S$ models.

We can think of $1-q_{1}$ as the cost of adjustment faced by holders of good cars. The lower the quality in the secondary market the greater the cost of adjustment, and the wider is the range of inaction, $(-\bar{z}, \bar{z})$. Since $q_{1} \geq \phi$, holders of lemons actually receive an adjustment subsidy. This is why they all adjust.

\subsubsection{Multiple Equilibria}

The monotonicity of $T_{F}$ is a reflection of the positive feedback in adjustment. If agents believe that the quality of cars in the secondary market has improved, then the range of inaction narrows, and quality in the secondary market improves. This strategic complementarity opens the possibility for multiple equilibria. A possibility that is confirmed by the following example. 
Example 1: Suppose that $F$ describes a discrete probability distribution on $\{-\hat{z}, \hat{z}\}$ where $\hat{z} \in\left[\sqrt{1-q_{0}}, \sqrt{1-\phi}\right)$. Then if agents believe that the average quality in the secondary market is $\phi$, no holder of a good car adjusts and the expected quality in the secondary market is $\phi$, whereas if agents believe that the average quality in the secondary market is $q_{0}$, all holders of good cars adjust and the expected quality in the secondary market is $q_{0}$.

\subsubsection{Comparative Statics}

In order to present the comparative statics it is useful to rule out multiple equilibria. Assumption 1 presents a sufficient condition for a unique equilibrium.

Assumption 1: $F$ has a density $f(z) . f(z)$ is symmetric and quasiconcave with $f(0)<\frac{1}{\sqrt{1-\phi}}\left(\frac{1-\pi}{\pi}\right)$.

The assumption prevents $F$ from placing too much weight on any open set. So that changes in the bands do not induce to large a change in market quality.

Lemma 1 Assumption 1 is sufficient for there to exist a unique equilibrium.

We wish to analyze the effect of an increase in the dispersion of the shock on the size of the $s S$ bands. Before presenting this result, we must first clarify what we mean by an "increase in dispersion". We borrow our definition of dispersion from Sargent [1987, p.64-65].

Definition 1 Consider two Borel probability measures $\mu$ and $\nu$ on $R$. Suppose that both $E_{\mu}[x]$ and $E_{\nu}[x]$ are well defined and finite, and that $E_{\mu}[x]=$ $E_{\nu}[x] \equiv \bar{x}$. Then $\nu$ has greater dispersion than $\mu$ iff $\forall x_{1}, x_{2}$ such that $x_{1} \leq \bar{x} \leq x_{2}$ we have

$$
\int_{-\infty}^{x_{1}} d \nu \geq \int_{-\infty}^{x_{1}} d \mu
$$

and

$$
\int_{x_{2}}^{+\infty} d \nu \geq \int_{x_{2}}^{+\infty} d \mu
$$


This is the natural definition of dispersion in the context of $s S$ adjustment where the main issue that we care about is whether or not a shock takes the agent outside the $s S$ bands. Intuitively, an increase in dispersion requires that the probability that the agent adjusts increases regardless of how the bands (about the mean) are defined. Notice that if $\nu$ that is more disperse than $\mu$, than it is also a mean preserving spread of $\mu .^{8}$

With this definition in hand, we have the following result

Proposition 2: With Assumption 1, an increase in dispersion leads to a reduction in $\bar{z}$.

Given any range of inaction $[-\bar{z}, \bar{z}]$, an increase in dispersion increases the probability that holders of good cars adjust. This adjustment leads to an increase in the average quality of cars in the secondary market, which drives down the lemons cost and causes the bands to narrow.

This effect is the opposite of what we normally observe in $s S$ models. Normally, an increase in variance, increases the option value to waiting and cause the range of inaction to widen. Since holders of used cars in our model have a one period horizon, the adjustment decision is essentially static. The standard effect therefore does not appear. In a model with more dynamics, such as the model of the next section, both effects would be present. In that case, we would not be able to sign the effect of an increase in the dispersion of the shock on the width of the $s S$ bands.

\subsubsection{Scrapping}

If the price of new cars is low enough, agents may strictly prefer purchasing new cars to used ones. In this case there will be excess supply in the used car market and $p_{1}$ will fall to zero. The value function becomes:

$$
V_{1}(x, z)=\max \left\{x-z^{2}+\beta V_{0}, V_{0}\right\}
$$

Note that all agents with lemons still adjust. These agents would prefer exchanging their used car for another used car because it improves their

\footnotetext{
${ }^{8}$ The converse does not necessarilty hold. Mean preserving spreads do not necessarily lead to greater adjustment in an $s S$ model. Suppose that $\mu$ places equal probability on the points $\{-1,1\}$, and that $\nu$ places probability one half on 0 and a quarter each on -2 and 2. Then $\nu$ is a mean preserving spread of $\mu$ but is not an increase in dispersion. If $x_{1}$ is taken to be -.5 then the first condition in the definition is violated.
} 
match. Since new cars are preferred to used cars, purchasing a new car clearly dominates holding on to their lemon. Agents with good cars adjust if $|z|>\bar{z}$ where

$$
\bar{z}=\sqrt{1-(1-\beta) V_{0}}
$$

In this case the number of agents with new cars will not remain at its initial value of one half. Let $N_{t}$ denote the number of agents purchasing new cars at date $t . N_{t}$ follows an autoregressive process

$$
N_{t}=\left(1-N_{t-1}\right)+[(1-\pi)+\alpha \pi] N_{t-1}
$$

Here the first term represents holders of used cars in period $t-1$ who purchase new cars in period $t$. The second term represents holders of new cars in period $t-1$, a proportion $1-\pi$ of which have lemons and adjust with probability one,

and a proportion $\pi$ have good cars and adjust with probability $\alpha=1-\int_{-\bar{z}}^{\bar{z}} d F$. It is easy to see that this process is stationary and converges to

$$
\bar{N}=\frac{1}{1+(1-\alpha) \pi} \geq \frac{1}{2} .
$$

\section{Dynamics}

We now extend the life of cars so that we may study how the bands evolve as cars age. We return to the two quality model of section 2, but assume that cars last for three periods. We consider two information structures. In the first the trading history is not observable, so that buyers cannot distinguish between two year old cars that have had two owners and those which have had only one owner. This version highlights the effect that depreciating match quality has on the time profile of the adjustment triggers. In the second information structure, trading history is observable. This version illustrates the role of trade history in signalling quality and the effect that this signalling has on the evolution of the adjustment triggers over time.

\subsection{Unobservable Trading Histories}

We retain the basic structure of Section 2, making alterations to allow for three period lived cars. First, if consumers cannot observe the history of ownership of the cars they buy, there will be three markets: one for new cars, one for one year old cars, and one for two year old cars. 
Second, we amend the matching function. As before $z=0$, during the first period that an agent owns a car whether or not the car is new or used. In the second period of ownership $z=\varepsilon_{1}$, where $\varepsilon_{1}$ is distributed on $\mathbb{R}$ according to the distribution $F$. In the third period of ownership the fit is given by $z=\varepsilon_{1}+\varepsilon_{2}$, where both $\varepsilon_{1}$ and $\varepsilon_{2}$ are independent draws from $F$.

To simplify the analysis, we assume that $F$ has a density $f$ which is symmetric about zero and everywhere positive on $\mathbb{R}$. The fact that $F$ has a density will allow us to use Brouwer's fixed point theorem to prove existence. Symmetry ensures that upward and downward adjustment are the same, and positivity ensures that some agents receive shocks so large that they adjust. This adjustment ensures that supply in all three markets is positive. In addition we will assume that the price of new cars is high enough that demand is positive as well. This ensures that all markets exist and saves us from cataloguing numerous special cases.

Agents choose whether or not to adjust and when they adjust they choose what type of car to buy. The optimal adjustment strategies are described by threshold levels of $z$ contingent on the age and quality of the car that the agent possesses. We look for a competitive equilibrium in which (1) the prices $p_{1}$ and $p_{2}$ clear the market for one and two year old cars respectively, (2) agents choose their thresholds optimally given prices and the expected qualities in the market for one and two year old cars, $q_{1}$ and $q_{2}$, (3) expectations are rational in that $q_{1}$ and $q_{2}$ are the average qualities of cars in the market for one and two year old cars.

As before we fix the price of new cars at $p_{0}$ and the expected quality of new cars at $q_{0}$.

\subsection{Solution}

We begin by characterizing the adjustment triggers as a function of the quality of cars in the used car market. Consider first the decision of the agent who enters period $t$ with a two year old car. Let $V_{2}$ denote the value of this agent's optimal policy. This value depends on the quality of the car which is known to be $x \in\{\phi, 1\}$ and on the match parameter $z$. Given these state variables, the agent chooses whether or not to sell the car and, if the choice is to sell, what type of car to purchase. As in the two period model, buyers will be indifferent between what type of car they buy in equilibrium. Without loss of generality, we equate the value of adjusting with the value of 
purchasing a two year old car. The value of an optimal policy is therefore:

$$
V_{2}(x, z)=\max \left\{x-z^{2}+\beta V_{0}, q_{2}+\beta V_{0}\right\}
$$

where $V_{0}$ is the value to purchasing a new car. The interpretation is essentially the same as in the one period model. The first term represents the value of holding on to the current car, whereas the second term represents the value of adjusting.

Next consider the decision of an agent who enters period $t$ with a one year old car. The quality of the car is known to be $x \in\{\phi, 1\}$ and the match parameter is $z$. In this case, the value of an optimal policy is

$$
V_{1}(x, z)=\max \left\{x-z^{2}+\beta E_{\varepsilon}\left[V_{2}(x, z+\varepsilon)\right], q_{1}+\beta E_{\left(x^{\prime}, \varepsilon\right)}\left[V_{2}\left(x^{\prime}, \varepsilon\right)\right]\right\}
$$

where $E_{\omega}$ represents the mathematical expectation with respect to the distribution of the random variable $\omega$. Note that $x^{\prime}$ is drawn from the equilibrium distribution of qualities in the market for one year old cars. Again the first term is the value of holding and the second term is the value of adjusting (this time to another one year old car). In this case, the agent must form expectations concerning the evolution of the match.

Since some holders of good cars receive terrible matches and adjust, the expected quality on the secondary market is above that of a lemon. Therefore all holders of lemons adjust in each period.

Lemma 2: In any equilibrium, all agents with lemons adjust in every period regardless of the age of their car.

Let $\bar{z}_{1}$ and $\bar{z}_{2}$ represent the adjustment thresholds for holders of good one and two year old cars. Equations (6) and (7) imply that an agent sells a good one year old car if $|z|>\left|\bar{z}_{1}\right|$ where

$$
\bar{z}_{1}=\sqrt{1-q_{1}+\beta E_{\left(x^{\prime}, \varepsilon\right)}\left[V_{2}\left(1, \bar{z}_{1}+\varepsilon\right)-V_{2}\left(x^{\prime}, \varepsilon\right)\right]} .
$$

and a good two year old car if $|z|>\left|\bar{z}_{2}\right|$ where

$$
\bar{z}_{2}=\sqrt{1-q_{2}} \text {. }
$$


Equations (8) and (9) determine the adjustment thresholds as functions of $q_{1}$ and $q_{2} \cdot{ }^{9}$ We now analyze the determination of these qualities. Consider first the market for one year old cars. Given $\bar{z}_{1}$, the total mass of agents with high quality cars who adjust is $2 \pi F\left(-\bar{z}_{1}\right)$. Hence the proportion of good cars is

$$
\lambda_{1}=\frac{2 \pi F\left(-\bar{z}_{1}\right)}{(1-\pi)+2 \pi F\left(-\bar{z}_{1}\right)} \leq \pi
$$

and the expected quality is,

$$
q_{1}=\lambda_{1}+\left(1-\lambda_{1}\right) \phi
$$

The situation is slightly more complex in the market for two year old used cars. Given $\bar{z}_{1}$, the distribution of matches for two year old cars is

$$
G_{\bar{z}_{1}}(z)=F(z)\left[2 F\left(-\bar{z}_{1}\right)\right]+\int_{\bar{z}_{1}}^{\bar{z}_{1}} F(z-\varepsilon) f(\varepsilon) d \varepsilon .
$$

The first term represents the $z$ 's of the agents who have held their car for one period. Their match parameter is described by a single draw from $F$. As they must have obtained their cars from agents who sold one year old cars there are $2 F\left(-\bar{z}_{1}\right)$ of them. The second term represents the $z$ 's of agents who have held their cars for two periods. Their match is represented by the sum of two draws from $F, \varepsilon_{1}+\varepsilon_{2}$. As these agents choose not to sell their cars last period, $\varepsilon_{1}$ lies between $-\bar{z}_{1}$ and $\bar{z}_{1}$. The density of these realizations is $f\left(\varepsilon_{1}\right)$. For $\varepsilon_{1}+\varepsilon_{2} \leq z$, it must be the case that $\varepsilon_{2} \leq z-\varepsilon_{1}$. This gives the second term.

Given $\bar{z}_{2}$ and $G_{\bar{z}_{1}}(z)$, the mass of agents with two year old good cars $2 \pi G_{\bar{z}_{1}}\left(-\bar{z}_{2}\right)$. Hence the proportion of good cars is

$$
\lambda_{2}=\frac{2 \pi G_{\bar{z}_{1}}\left(-\bar{z}_{2}\right)}{(1-\pi)+2 \pi G_{\bar{z}_{1}}\left(-\bar{z}_{2}\right)} \leq \pi,
$$

and the expected quality is

\footnotetext{
${ }^{9}$ It is not immediately obvious that (8) determines $\bar{z}_{1}$ since $V_{2}$ appears in the equation. However, given $q_{2}$ and $\bar{z}_{1}$ we can evaluate

$$
V_{2}\left(1, \theta \bar{z}_{1}+\varepsilon\right)-V_{2}\left(x^{\prime}, \varepsilon\right)=\max \left\{1-\bar{z}_{1}^{2}, q_{2}\right\}-\max \left\{x^{\prime}-z^{2}, q_{2}\right\} .
$$

Hence the right hand side of (8) is a function of $q_{1}, q_{2}$ and $\bar{z}_{1}$. Moreover, the right hand side is countinuously and monotonically decreasing in $\bar{z}_{1}$. Hence there exists a unique $\bar{z}_{1}$ that satisfies (8) given $q_{1}$ and $q_{2}$.
} 


$$
q_{2}=\lambda_{2}+\left(1-\lambda_{2}\right) \phi
$$

In order to prove existence and analyze the properties of equilibrium, it is useful to construct the mapping $T:[\phi, 1] \times[\phi, 1] \rightarrow[\phi, 1] \times[\phi, 1]$ as follows. Note that given $\left(q_{1}, q_{2}\right) \in[\phi, 1] \times[\phi, 1]$, equations (8) and (9) pin down $\bar{z}_{1}$ and $\bar{z}_{2}$. Then given $\bar{z}_{1}$ and $\bar{z}_{2}$, equations (10) and (12) pin down $\lambda_{1}$ and $\lambda_{2}$. Finally given $\lambda_{1}$ and $\lambda_{2}$, equations (11) and (13) define $q_{1}^{\prime}, q_{2}^{\prime} \in[\phi, 1] \times[\phi, 1]$. We set $T\left(q_{1}, q_{2}\right)=\left(q_{1}^{\prime}, q_{2}^{\prime}\right)$. The equilibrium values of $q_{1}$ and $q_{2}$ arise as fixed points of $T$.

Existence of an equilibrium $q_{1}$ follows from the continuity of $T$ and Brouwer's fixed point theorem.

Proposition 3: There exists an equilibrium in the model with unobservable trading histories.

Given the equilibrium qualities and adjustment triggers, we can solve for the equilibrium prices. We first calculate the value of a new car. $V_{0}$ must satisfy

$$
V_{0}=q_{0}+\beta E\left\{V_{1}(x, \varepsilon)\right\}-p_{0}
$$

Note given $q_{1}$ and $q_{2}$, the existence of a solution $V_{0}$ to (14) follows from standard dynamic programming arguments.

The utility from purchasing in the new car market must be the same as purchasing in the two year old car market:

$$
V_{0}=q_{2}-p_{2}+\beta V_{0}
$$

This pins down $p_{2}$ :

$$
p_{2}=q_{2}-(1-\beta) V_{0}
$$

Similarly, the utility from purchasing in the new car market must be the same as purchasing a one year old car:

$$
V_{0}=E\left[x+\beta\left(\max \left\{V_{0}+p_{2}, x-\varepsilon^{2}+\beta V_{0}\right\}\right)\right]-p_{1}
$$

This pins down $p_{1}$ :

$$
p_{1}=E\left[x+\beta\left(\max \left\{V_{0}+p_{2}, x-\varepsilon^{2}+\beta V_{0}\right\}\right)\right]-V_{0}
$$




\subsection{Evolution of the Bands}

Comparing $\bar{z}_{1}$ and $\bar{z}_{2}$, we have

$$
\bar{z}_{1}^{2}-\bar{z}_{2}^{2}=q_{2}-q_{1}+\beta E_{\left(x^{\prime}, \varepsilon\right)}\left[V_{2}\left(1, \bar{z}_{1}+\varepsilon\right)-V_{2}\left(x^{\prime}, \varepsilon\right)\right] .
$$

The sign of this expression depends on the balance between three forces. The first force is the accumulation of shocks, which causes the distribution of the match parameter to become more disperse over time. The following lemma shows that $G_{\bar{z}_{1}}$ is more disperse than $F$.

Lemma 3: For any $\bar{z}_{1}>0$, the distribution $G_{\bar{z}_{1}}$ is more disperse than $F$.

The implication of this lemma is that there will be more adjustment in the second period for any given adjustment threshold. This increase in dispersion tends to make $q_{2}$ larger than $q_{1}$. Due to the complementarity, this force tends to make the bands shrink over time. We call this effect the "dispersion effect".

Unfortunately, this alone is not enough to $\operatorname{sign} \bar{z}_{1}^{2}-\bar{z}_{2}^{2}$. The last term on the right-hand side of equation (15) also plays a role. This term represents the trade-off between entering the last period with a good car and entering the last period with a good match. The following example makes this tradeoff clear.

Example 2: Suppose that $F$ is mean zero, has mass $\delta$ at 0 , and a mass $1-\delta$ distributed uniformly over a range greater than $2 \max \left\{\bar{z}_{1}, \bar{z}_{2}\right\}$.

This distribution has the property that being close to an adjustment trigger does not increase the probability of subsequent adjustment. Given any adjustment trigger, $\bar{z}$, and any initial position $z \in[-\bar{z}, \bar{z}]$, the probability that a shock to $z$ results in a new match outside of $[-\bar{z}, \bar{z}]$ is independent of the initial position $z$. Hence if $\bar{z}_{1}=\bar{z}_{2}=\bar{z}$, then $q_{1}=q_{2}$. This neutralizes the effect that dispersion has on the incentive to adjust.

We now consider two special cases of this example. We begin by setting $\delta$ equal to zero. ${ }^{10}$ In this case the expected value of a car tomorrow is independent of its match today. Consider an agent with match $z \in\left[-\bar{z}_{1}, \bar{z}_{1}\right]$. In the next period the match will be $z+\varepsilon$ where $\varepsilon$ is drawn from $F$. Since

\footnotetext{
${ }^{10}$ Danziger (1999) has used this distrubutional assumption to simplify the aggregation of a dynamic $s S$ model.
} 
$F$ is uniform with support greater than $2 \max \left\{\bar{z}_{1}, \bar{z}_{2}\right\}$, the probability of adjusting is independent of $z$. Moreover, the distribution of $z+\varepsilon$, conditional on $z+\varepsilon \in\left[-\bar{z}_{2}, \bar{z}_{2}\right]$, is uniform. Hence $E_{\left(x^{\prime}, \varepsilon\right)} V_{2}\left(x^{\prime}, z+\varepsilon\right)$ is independent of $z$. It follows that

$$
E_{\left(x^{\prime}, \varepsilon\right)}\left[V_{2}\left(1, \bar{z}_{1}+\varepsilon\right)-V_{2}\left(x^{\prime}, \varepsilon\right)\right]>E_{\left(x^{\prime}, \varepsilon\right)}\left[V_{2}\left(x^{\prime}, \bar{z}_{1}+\varepsilon\right)-V_{2}\left(x^{\prime}, \varepsilon\right)\right]=0 .
$$

where the inequality follows from the fact that it is always better to have a good car than a car selected randomly off the used car market. Equation (15) then implies $\bar{z}_{1}>\bar{z}_{2}$. In this case, there is an advantage to having a good car, but no advantage to having a good match. We call the effect that holding a good car has on non-adjustment the "bird-in-hand effect".

We will now present a numerical example in which $z_{1}<z_{2}$. Take $\delta=\pi=$ $\phi=.5$ and $\beta=.95$ and let the uniform component of $F$ be given by the density $u=.05$ over the range $[-10,10]$. Given this parameterization, the unique equilibrium is given by $\bar{z}_{1}=.5548$ and $\bar{z}_{2}=.5830$. In the equilibrium,

$$
E_{\left(x^{\prime}, \varepsilon\right)}\left[V_{2}\left(1, \bar{z}_{1}+\varepsilon\right)-V_{2}\left(x^{\prime}, \varepsilon\right)\right]=-.0335
$$

so that the benefit of entering the next period with a good match outweighs the benefit of entering with a good car. The features of the parameterization that contribute to this result are a very wide uniform component of $F$ and an intermediate value of $\delta . \delta>0$ implies that there is some persistence to the match. $\delta<1$ and the wide uniform ensure that there is a significant amount of adjustment. Together, these imply that if the agent holds onto the good car today, they will either adjust tomorrow or they will have a bad match tomorrow. On the other hand, if they adjust today they will get a good match tomorrow and potentially a good car. We call the motivation to adjust to get a better match the "matching effect".

Which of these effects is most likely to be important? The dispersion effect is likely to dominate in practice. It took a lot of effort to make the matching effect dominate. First, we chose a shock process that eliminated the dispersion effect. Second, we required the process to have the right mix of persistence and dispersion. The matching effect requires both that a good match is valuable and that a good car is not. Persistence alone increases the value of a good match but also reduces trade and therefore reduces the probability of getting a good car in the secondary market. High dispersion increases trade but reduces the value of a good match.

There is also a sense in which the bird-in-hand and matching effects are artifacts of the two period model. Consider a version of Example 2 in which 
cars, instead of depreciating after two periods, broke down at the end of a each period with a fixed probability $\chi$. The value of a $s$ year old car of quality $x$ with a match $z$ becomes

$$
V_{s}(x, z)=\max \left\{\begin{array}{c}
1-z^{2}+\chi \beta V_{0}+(1-\chi) \beta E_{\varepsilon} V_{s+1}(x, z+\varepsilon), \\
q_{s}+\chi \beta V_{0}+(1-\chi) \beta E_{\left(x^{\prime}, \varepsilon\right)} V_{s+1}\left(x^{\prime}, \varepsilon\right)
\end{array}\right\} .
$$

All variables have the same interpretation, except that now cars survive with probability $(1-\chi)$. In this environment there is an equilibrium in which the value of a used car is independent of its age and the adjustment triggers are also be independent of the age of the car. Given constant triggers, the distributional assumption ensures constant quality in the secondary market. With $q_{s}=q$, the $V_{s}$ are independent of $s$ and the triggers are constant. In the examples above, the bird-in-hand and matching effects had an effect on $\bar{z}_{1}$, but not $\bar{z}_{2}$ since cars died at the end of the second period. We conjecture that these effects are related to the increasing probability that a car breaks down as it ages.

\subsection{Observable Trading Histories}

If the trading history of a car is observable to the buyers, there will be four markets: new cars, one year old used cars, two year old cars/original owner, and two year old cars/new owner. We will refer to the market for two year old cars with original owners as the $O 2$ market and refer to the market for two year old cars with new owners as the $N 2$ market. The prices corresponding to each market will be denoted $\left\{p_{0}, p_{1}, p_{O 2}, p_{N 2}\right\}$.

We solve the model backwards, beginning with two year old cars. The reasoning is similar to the model of section 2. Given $j \in\{O, N\}$, the value functions are

$$
V_{j 2}(x, z,)=\max \left\{x-z^{2}+\beta V_{0}, q_{j 2}+\beta V_{0}\right\} .
$$

As before holders of lemons always choose to adjust and the adjustment triggers for holders of good cars are given by

$$
\bar{z}_{j 2}=\sqrt{1-q_{j 2}}
$$

Working backwards gives us the value of owning a one year old car, of quality $z$ with current shock $x$ as:

$$
V_{1}(x, z)=\max \left\{x-z^{2}+\beta E_{\varepsilon}\left[V_{O 2}(x, z+\varepsilon)\right], q_{1}+\beta E_{x^{\prime}, \varepsilon}\left[V_{N 2}\left(x^{\prime}, z+\varepsilon\right)\right]\right\}
$$


It is no longer the case that agents holding lemons that are one year old will necessarily adjust. It may be more profitable to enter the second period with a two year old car that is first hand. We therefore must calculate triggers for both types of car. The trigger for high quality car owners is now:

$$
\bar{z}_{1}=\sqrt{1-q_{1}+\beta\left[E_{\varepsilon} V_{O 2}(1, z+\varepsilon)-E_{x^{\prime}, \varepsilon} V_{N 2}\left(x^{\prime}, \varepsilon\right)\right]} .
$$

Let $\hat{z}_{1}$ denote the adjustment trigger for holders of one year old lemons. Then we have,

$$
\hat{z}_{1}=\max \left\{0, \sqrt{\phi-q_{1}+\beta\left[E_{\varepsilon} V_{O 2}(\phi, z+\varepsilon)-E_{x^{\prime}, \varepsilon} V_{N 2}\left(x^{\prime}, \varepsilon\right)\right]}\right\} .
$$

Within the set of two year old cars with original owners, let $\pi_{O}$ denote the fraction that are good cars. Let $\pi_{N}$ denote the corresponding proportion for new owners. $\pi_{O}$ and $\pi_{N}$ are functions of $\bar{z}_{1}$ and $\hat{z}_{1}$ :

$$
\begin{gathered}
\pi_{O}=\frac{\pi \int_{-\bar{z}_{1}}^{\bar{z}_{1}} d F}{(1-\pi) \int_{-\hat{z}_{1}}^{\hat{z}_{1}} d F+\pi \int_{-\bar{z}_{1}}^{\bar{z}_{1}} d F} \\
\pi_{N}=\frac{\left(1-\int_{-\bar{z}_{1}}^{\bar{z}_{1}} d F\right) \pi}{\left(1-\int_{-\hat{z}_{1}}^{\hat{z}_{1}} d F\right)(1-\pi)+\left(1-\int_{-\bar{z}_{1}}^{\bar{z}_{1}} d F\right) \pi}
\end{gathered}
$$

Given $\pi_{O}$ and $\pi_{N}$, we can calculate the proportion of good cars sold on each second year market:

$$
\begin{aligned}
\lambda_{N 2}= & \frac{\left(1-\int_{-\bar{z}_{N 2}}^{\bar{z}_{N 2}} d F\right) \pi_{j}}{1-\pi_{j} \int_{-\bar{z}_{N 2}}^{\bar{z}_{N 2}} d F} \\
\lambda_{O 2}= & \frac{\left(1-\int_{-\bar{z}_{O 2}}^{\bar{z}_{O 2}} d G_{\bar{z}_{1}}\right) \pi_{j}}{1-\pi_{j} \int_{-\bar{z}_{O 2}}^{\bar{z}_{O 2}} d G_{\bar{z}_{1}}}
\end{aligned}
$$

The integral is with respect to $F$ in the case of $\lambda_{N 2}$ since all of the participants in this market are new owners and hence have perfect matches in the previous period. From this we calculate the average quality in each second year market

$$
q_{j 2}=\lambda_{j 2}+\left(1-\lambda_{j 2}\right) \phi
$$


Returning to the one year old market, the proportion of good cars sold is $\pi_{N}$ and the expected quality in the market is

$$
q_{1}=\pi_{N}+\left(1-\pi_{N}\right) \phi
$$

Finally, $V_{0}$ must satisfy:

$$
V_{0}=q_{0}+\beta E_{0} V_{1}(x, z)-p_{0}
$$

\subsubsection{Properties of equilibrium}

Since $\phi<1$ and $V_{O 2}(1, z+\varepsilon)>V_{O 2}(\phi, z+\varepsilon)$, it follows immediately that $\bar{z}_{1}>\hat{z}_{1}$. Holders of one year old lemons are more likely to adjust than holders of one year old good cars. The fact that holders of lemons are more likely to adjust after one year means that a higher proportion of owners in the $O 2$ hold good cars as compared to the $N 2$ market, $\pi_{O}>\pi_{N}$. Given that $\pi_{O}>\pi_{N}$ and that $G_{\bar{z}_{1}}$ is more dispersed than $F$, there is more adjustment by owners of good cars in the $O 2$ market. This implies that $\bar{z}_{O 2}<\bar{z}_{N 2}, \lambda_{O 2}>\lambda_{N 2}$, and $q_{O 2}>q_{N 2}$. The quality of cars transacted in the original owners market is greater than the quality of cars transacted in the new owners market and this makes original holders of good cars more likely to adjust.

We can compare the equilibrium with observable trading histories with the equilibrium with unobservable trading history. First, $\bar{z}_{1}$ is larger when history is observable. The value of holding is larger because its signals that the car is good and the return to adjusting is lower because the agent enters the N2 market with all the lemons. Together with the fact that $\hat{z}_{1}$ is nonnegative implies that there is less trade in one year old cars when history is observable. Second, since an increase in $\bar{z}_{1}$ increases the dispersion of $G_{\bar{z}_{1}}$, the distribution of matches is more dispersed in the $O 2$ market than in the market for two year old cars without observable trading histories. This together with the fact that $\pi_{O}>\pi$ implies $\bar{z}_{O 2}<\bar{z}_{2}$. Finally, $\pi_{N}<\pi$ and $G_{\bar{z}_{1}}$ is more dispersed than $F$ imply $\bar{z}_{2}<\bar{z}_{N 2}$.

In the model with unobservable trading history the only misallocation relative to perfect information was that some agents with good cars failed to trade. In the model with observable history there is an additional misallocation in the one year old market. Some of the agents with lemons decide not to trade. The presence of signalling introduces an additional opportunity cost to adjustment for all agents. In the second period, however, there may be a gain in efficiency. As agents learn which cars are good and which are bad, the adverse selection problem is reduced and trade incidence increases. 


\section{Empirical Implications}

One empirical application regards the estimation of depreciation from market prices. If the adverse selection problem is initially severe, then the initial fall in price reflects a combination of depreciation and adverse selection. Physical depreciation is therefore overestimated initially. If adverse selection becomes less severe as cars age, then subsequent changes in price reflect both depreciation and the easing of adverse selection. Physical depreciation is therefore underestimated in later years.

Another application concerns the effect of an increase in the variance of the match parameter on the width of the $s S$ bands. Eberly [1994], for example, regresses the size of the $s S$ bands on the variance of an individual's income. She finds a mildly positive coefficient on income variance. To the extent that individual income variance is purely idiosyncratic, her estimate of the traditional $s S$ model is unbiased. If, however, individual income variance is correlated in some way with the variance of shocks to the market, because, for example, agents with high income variance tend to purchase similar cars, her estimates mix the traditional $s S$ effect and the thick market effect that arises from adverse selection. Her estimate of the traditional $s S$ effect is downward biased.

\section{Conclusion}

The used car market features so prominently in both the literature on adverse selection and in the literature on $s S$ adjustment that it is surprising that existing models of this market have not, to this date, incorporated both features. We presented a model in which $s S$ adjustment arose from an adverse selection problem. The presence of adverse selection creates a complementarity between agents adjustment decisions. This has several implications for the nature of the equilibrium $s S$ policies. As the variance of the shock process increases more agents adjust and the $s S$ bands shrink. As the car ages, matches deteriorate, more adjustment takes place and the $s S$ bands tend to shrink. 


\section{Appendix A: Proofs of the Propositions}

Proposition 1: $T_{F}$ is non-decreasing, upper semi-continuous, and has the fixed point property.

Proof: Consider $q_{1}, \hat{q}_{1} \in[\phi, 1]$ with $q_{1}<\hat{q}_{1}$. It follows from (1) that $\bar{z}\left(q_{1}\right)>\bar{z}\left(\hat{q}_{1}\right)$. It follows from $(2)$ that $\lambda_{F}\left(\bar{z}\left(q_{1}\right)\right) \leq \lambda_{F}\left(\bar{z}\left(\hat{q}_{1}\right)\right)$ (the inequality is strict if $F$ places positive probability on the set $\left[-\bar{z}\left(\hat{q}_{1}\right),-\bar{z}\left(q_{1}\right)\right) \cup$ $\left.\left(\bar{z}\left(q_{1}\right), \bar{z}\left(\hat{q}_{1}\right)\right]\right)$. It follows from $(3)$ that $T_{F}\left(q_{1}\right) \geq T_{F}\left(\hat{q}_{1}\right) . T_{F}\left(q_{1}\right)$ is therefore non-decreasing in $q_{1}$. We conclude that a fixed point exists by Tarsky's fixed point theorem. Upper semi-continuity follows from our assumption that, when indifferent, agents adjust.

Lemma 1 Assumption 1 is sufficient for there to exist a unique equilibrium..

Proof: Combining equations (1), (2), and (3), we construct the mapping $\bar{z} \rightarrow \bar{z}^{\prime}$ defined by

$$
\bar{z}^{\prime}=\sqrt{\frac{(1-\pi)(1-\phi)}{1-\pi+2 \pi F(-\bar{z})}} .
$$

Any equilibrium cutoff is a fixed point of this mapping. Consider

$$
\begin{aligned}
\frac{d \bar{z}^{\prime}}{d \bar{z}} & =\sqrt{\frac{(1-\pi)(1-\phi)}{1-\pi+2 \pi F(-\bar{z})}} \frac{\pi f(-\bar{z})}{1-\pi+2 \pi F(-\bar{z})} \\
& \leq \frac{\pi}{1-\pi} f(-\bar{z}) \sqrt{1-\phi} .
\end{aligned}
$$

The assumption implies $\frac{d \bar{z}^{\prime}}{d \bar{z}}<1$ which proves the lemma. $\square$

Proposition 2: With Assumption 1, an increase in dispersion leads to an increase in $\bar{z}$.

Proof: Consider two densities $f$ and $g$ which have the same mean. Suppose that $f$ is more disperse than $g$ in the sense of definition 1 . Consider $q_{1} \in[\phi, 1]$. Let $\bar{z}\left(q_{1}\right)$ be defined by (1). Since $f$ is more disperse than $g$, $\int_{-\bar{z}\left(q_{1}\right)}^{\bar{z}\left(q_{1}\right)} g(z) d z \geq \int_{-\bar{z}\left(q_{1}\right)}^{\bar{z}\left(q_{1}\right)} f(z) d z$ which implies that $\lambda_{g}\left(\bar{z}\left(q_{1}\right)\right) \leq \lambda_{f}\left(\bar{z}\left(q_{1}\right)\right)$. It follows that $T_{g}\left(q_{1}\right) \leq T_{f}\left(q_{1}\right)$ for all $q_{1}$. Moreover, since $T_{f}$ is monotonically increasing in $q_{1}$, the unique fixed point under $f$ must lie in the interval $\left(q_{1}, 1\right)$. 
Lemma 2: In any equilibrium, all agents with lemons adjust in every period regardless of the age of their car.

Proof: Consider first holders of two year old lemons. They choose between $q_{2}+\beta V_{0}$ if they adjust now or $\phi-z^{2}+\beta V_{0}$ if they hold on to the car. Since $q_{2} \geq \phi$ they adjust. Now consider holders of one year old lemons. They receive $q_{1}+\beta E_{(x, \varepsilon)}\left[V_{2}(x, \varepsilon)\right]$ if they adjust and $\phi-z^{2}+\beta E_{\varepsilon}\left[V_{2}(\phi, z+\varepsilon)\right]$ if they hold. Since holders of two year old lemons adjust, $V_{2}(\phi, z+\varepsilon)=q_{2}+\beta V_{0}$. It follows from $(6)$ that $E_{(x, \varepsilon)}\left[V_{2}(x, \varepsilon)\right] \geq q_{2}+\beta V_{0}$. Since $q_{2} \geq \phi$, they adjust. $\square$

Proposition 3: There exists an equilibrium in the model with unobservable trading histories.

Proof: Consider $T:[\phi, 1] \times[\phi, 1] \rightarrow[\phi, 1] \times[\phi, 1]$ as defined above. Each of the component mappings is continuous and differentiable. The only questionable case is (8) which gives $\bar{z}_{1}$ as a function of $q_{1}$ and $q_{2}$. But note that $\beta E_{\left(x^{\prime}, \varepsilon\right)}\left[V_{2}\left(1, \bar{z}_{1}+\varepsilon\right)-V_{2}\left(x^{\prime}, \varepsilon\right)\right]$ is continuous and differentiable in $\bar{z}_{1}$ and $q_{1}$ so that this mapping is continuous and differentiable by the implicit function theorem. We therefore have existence by Brouwer's fixed point theorem. $\square$

Lemma 3: For any $\bar{z}_{1}>0$, the distribution $G_{\bar{z}_{1}}$ is more disperse than $F$.

Proof: We will show that for $z \leq 0, G_{\bar{z}_{1}}(z) \geq F(z)$; a similar argument will apply to $z \geq 0$. Consider $\frac{G_{\bar{z}_{1}}(z)}{F(z)}=2 F\left(-z_{1}\right)+\int_{-z_{1}}^{z_{1}} \frac{F\left(z-\varepsilon_{1}\right)}{F(z)} f\left(\varepsilon_{1}\right) d \varepsilon_{1}$ for a $z \leq 0$. Note that our assumptions on $f$ imply that $F$ is convex over the interval $(-\infty, 0]$. Rewrite the integral as:

$$
\int_{-z_{1}}^{z_{1}} \frac{F\left(z-\varepsilon_{1}\right)}{F(z)} f\left(\varepsilon_{1}\right) d \varepsilon_{1}=\int_{0}^{z_{1}} \frac{F\left(z+\varepsilon_{1}\right)+F\left(z-\varepsilon_{1}\right)}{F(z)} f\left(\varepsilon_{1}\right) d \varepsilon_{1}
$$

Since $F$ is convex, we can apply Jensen's inequality to get

$$
\frac{1}{2}\left[F\left(z+\varepsilon_{1}\right)+F\left(z-\varepsilon_{1}\right)\right] \geq F(z)
$$

so that:

$$
\int_{-z_{1}}^{z_{1}} \frac{F\left(z-\varepsilon_{1}\right)}{F(z)} f\left(\varepsilon_{1}\right) d \varepsilon_{1} \geq \int_{0}^{z_{1}} \frac{2 F(z)}{F(z)} f\left(\varepsilon_{1}\right) d \varepsilon_{1}=2\left[F\left(z_{1}\right)-F(0)\right]
$$

Finally use the symmetry of $z_{1}$ and the fact that $F(0)=\frac{1}{2}$ to get:

$$
\frac{G_{\bar{z}_{1}}(z)}{F(z)} \geq 2 F\left(-z_{1}\right)+2\left[1-F\left(-z_{1}\right)-\frac{1}{2}\right]=1
$$

So that $G_{\bar{z}_{1}}(z) \geq F(z)$ for any $z \leq 0$. Thus $G_{\bar{z}_{1}}$ is more disperse than $F$. $\square$ 


\section{Appendix B: Comparative Statics with Mul- tiple Equilibria}

Let $\mathfrak{E}_{\mu}$ be the set of fixed points of $T_{\mu}$. We know from proposition 1 that this set is non-empty. It is easy to show that $\mathfrak{E}_{\mu}$ has a maximal and a minimal element. ${ }^{11} \underline{e}_{\mu}, \bar{e}_{\mu} \in \mathfrak{E}_{\mu}$ be the least and the greatest elements in $\mathfrak{E}_{\mu}$ respectively. Our first comparative static result concerns the effect of an increase in the dispersion of $z$ 's, on $\underline{e}_{\mu}$ and $\bar{e}_{\mu}$.

Our first result is that in increase in dispersion lifts the set of equilibrium qualities in the secondary market.

Proposition 2 Consider Borel measures $\mu$ and $\nu$ with the same finite mean and suppose that $\nu$ is more disperse than $\mu$. Then $\underline{e}_{\nu}>\underline{e}_{\mu}$ and $\bar{e}_{\nu}>\bar{e}_{\mu}$.

Proof: Consider $q_{1} \in[\phi, 1]$. Let $\bar{z}\left(q_{1}\right)$ be denied by $(1)$ and $\lambda_{\mu}(\bar{z})$ be defined by (2). Since $\nu$ is more disperse than $\mu, \int_{-\bar{z}\left(q_{1}\right)}^{\bar{z}\left(q_{1}\right)} d \mu(z) \geq \int_{-\bar{z}\left(q_{1}\right)}^{\bar{z}\left(q_{1}\right)} d \nu(z)$ which implies that $\lambda_{\mu}\left(\bar{z}\left(q_{1}\right)\right) \leq \lambda_{\nu}\left(\bar{z}\left(q_{1}\right)\right)$. It follows that $T_{\mu}\left(q_{1}\right) \leq T_{\nu}\left(q_{1}\right)$ for all $q_{1}$. Moreover, $T_{\mu}$ monotonically increasing in $q_{1}$, and is therefore continuous in $q_{1}$ except for upward jumps. These two properties enable us to apply Corollary 1 in Milgrom and Roberts [1994] which completes the proof. $\square$

Intuitively this theorem says that, as a whole, the set of equilibria rises as the trade shock becomes more and more disperse. Clearly if the equilibrium is unique then increases in dispersion cause increases the equilibrium $q_{1}$.

It is not the case that an increase in dispersion raises all elements in $\mathfrak{E}_{\mu}$. Generally, there are two types of equilibria depending on the slope of $T_{\mu}$ at the fixed point. For $e \in \mathfrak{E}_{\mu}$ that are of the first type, we have $T_{\mu}^{\prime}(e)<1$. In this case, a small increase in dispersion raises $e .{ }^{12}$ This class includes $\underline{e}_{\mu}$ and $\bar{e}_{\mu}$ if they are interior to $[\phi, 1]$.

\footnotetext{
${ }^{11}$ The existence of a minimal element follows directly from the upper semi-continuity and monotonicity of $T_{\mu}$. The existence of a maximal element follows from the monotonity of $T_{\mu}$ and the compactness of $[\phi, 1]$.

12 Technically, we can define a small increase in dispersion as follows. The space $\mathfrak{P}$ of Borel probability distributions on $\mathbb{R}$ is a metric space (Billingsley [1968]). Consider a closed subset $\mathfrak{P}$ whose elements have the same finite mean and are indexed by a parameter $\sigma$ such that higher levels of $\sigma$ imply greater dispersion. The normals with mean zero and variance $\sigma$ are one such subset. We can then associate a small increase in dispersion, with a small increase in $\sigma$.
} 
The second generic class of equilibria are characterized by $T_{\mu}^{\prime}(e)>1$. In this case a small increase in dispersion reduces $e$. $T_{\mu}^{\prime}\left(q_{1}\right)$ is greater than 1 when there are a large number of $z$ 's massed about $\pm \bar{z}\left(q_{1}\right)$. In this case, a small increase in $q_{1}$, leads to a large increase in $\lambda_{\mu}$, and a large increase in $T_{\mu}\left(q_{1}\right)$. There is a sense in which this class of equilibria are unstable to perturbations. A small change in $q_{1}$ from any of these points, if repeatedly passed through the mapping $T_{\mu}$ will eventually converge to an equilibrium of the first type. 


\section{References}

Abel, Andrew, Avinash Dixit, Janice Eberly, and Robert Pindyck, 1996, "Options, the Value of Capital, and Investment," Quarterly Journal of Economics 111, 753-778.

Adda, Jerome, and Russell Cooper, 1999, "Balladurette and Jeppette: A Discrete Approach", mimeo.., Boston University.

Akerlof, George, 1970, "The Market for 'Lemons': Quality Uncertainty and the Market Mechanism," Quarterly Journal of Economics, 84, 488500 .

Arrow, K., T. Harris, and J. Marschak, 1951, "Optimal Inventory Policy," Econometrica, 19, 205-272.

Blouin, Max, 1998, "Overlapping Generations of Cars," Brown University working paper.

Bar-Ilan, Avner, and Alan Blinder, 1992, "Evidence on the Optimality of Usually Doing Nothing," Journal of Money, Credit and Banking, 24, $258-272$.

Bertola, Giuseppe, and Ricardo Caballero, 1990, "Kinked Adjustment Costs and Aggregate Dynamics," NBER Macroeconomics Annual 1990, Cambridge: MIT Press.

Billingsley, Patrick, 1968, Convergence of Probability Measures, New York: Wiley \& Sons.

Caballero, Ricardo, 1993, "Durable Goods: An Explanation for their Slow Adjustment," Journal of Political Economy, 101, 351-384.

Caballero, Ricardo, 1994, "Notes on the Theory and Evidence on Aggregate Purchases of Durable Goods," Oxford Review of Economic Policy, 10, 107-117.

Caplin, Andrew, and John Leahy, 1999, "Durable Goods Cycles," NBER Working Paper No. 6987. 
Carroll, Christopher, and Wendy Dunn, 1997, "Unemployment Expectations, Jumping (S, s) Triggers and Household Balance Sheets," NBER Macroeconomics Annual 1997, Cambridge: MIT Press.

Danziger, Lief, 1999, "A Dynamic Economy with Costly Price Adjustments", American Economic Review, 89, 878-901.

Eberly, Janice, 1994, “Adjustment of Consumers' Durables Stocks: Evidence from Automobile Purchases," Journal of Political Economy, 102, 403-436.

Eisfeldt, Andrea, 1999, "Endogenous Liquidity in Asset Markets," University of Chicago mimeo..

Grossman, Sanford, and Guy Laroque, 1990, "Asset Pricing and Optimal Portfolio Choice in the Presence of Illiquid Durable Consumption Goods," Econometrica 58, 25-51.

Hendel, Igal, and Alessandro Lizzeri, 1999, "Adverse Selection in Durable Goods Markets," American Economic Review, 89, 1097-1115.

Leahy, John, and Joseph Zeira, 1999, "The Timing of Purchases and Aggregate Fluctuations," Boston University mimeo.

Milgrom, Paul, and John Roberts, 1994, "Comparing Equilibria," American Economic Review, 84, 441 - 59.

Sargent, Thomas, 1987, Dynamic Macroeconomic Theory, Cambridge: Harvard University Press.

Stiglitz, Joseph, 1997, Economics, New York: W. W. Norton \& Co..

Stolyarov, Dmitriy, 1999, "Stationary Equilibrium in Secondary Markets with Transaction Costs: Holding Patterns for Durable Goods," University of Michigan working paper. 